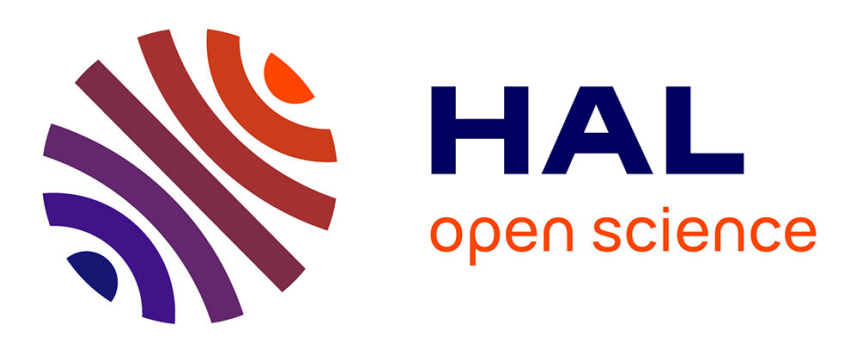

\title{
Agréables différends. L'esthétique galante de la querelle dans deux dialogues de Jean Chapelain et Jean-François Sarasin
}

Marine Roussillon

\section{- To cite this version:}

Marine Roussillon. Agréables différends. L'esthétique galante de la querelle dans deux dialogues de Jean Chapelain et Jean-François Sarasin. Littératures classiques, 2013, Le temps des querelles, 81, pp.51-62. 10.3917/licla.081.0051 . halshs-01487456

\section{HAL Id: halshs-01487456 \\ https://shs.hal.science/halshs-01487456}

Submitted on 12 Mar 2017

HAL is a multi-disciplinary open access archive for the deposit and dissemination of scientific research documents, whether they are published or not. The documents may come from teaching and research institutions in France or abroad, or from public or private research centers.
L'archive ouverte pluridisciplinaire HAL, est destinée au dépôt et à la diffusion de documents scientifiques de niveau recherche, publiés ou non, émanant des établissements d'enseignement et de recherche français ou étrangers, des laboratoires publics ou privés. 
« AGRÉABLES DIFFÉRENDS ». L'ESTHÉTIQUE GALANTE DE LA QUERELLE DANS DEUX DIALOGUES DE JEAN CHAPELAIN ET JEANFRANÇOIS SARASIN

Marine Roussillon

Armand Colin | « Littératures classiques »

2013/2 Nº $81 \mid$ pages 51 à 62

ISSN 0992-5279

ISBN 9782200928599

Article disponible en ligne à l'adresse :

http://www.cairn.info/revue-litteratures-classiques1-2013-2-page-51.htm

\section{Pour citer cet article :}

Marine Roussillon, « " Agréables différends ». L'esthétique galante de la querelle dans deux dialogues de Jean Chapelain et Jean-François Sarasin », Littératures classiques 2013/2 ( $\left.\mathrm{N}^{\circ} 81\right)$, p. 51-62.

DOI 10.3917/licla.081.0051

Distribution électronique Cairn.info pour Armand Colin.

(C) Armand Colin. Tous droits réservés pour tous pays.

La reproduction ou représentation de cet article, notamment par photocopie, n'est autorisée que dans les limites des conditions générales d'utilisation du site ou, le cas échéant, des conditions générales de la licence souscrite par votre établissement. Toute autre reproduction ou représentation, en tout ou partie, sous quelque forme et de quelque manière que ce soit, est interdite sauf accord préalable et écrit de l'éditeur, en dehors des cas prévus par la législation en vigueur en France. Il est précisé que son stockage dans une base de données est également interdit. 


\title{
Marine Roussillon
}

\author{
«AGRÉABLES DIFFÉRENDS》. \\ L'ESTHÉTIQUE GALANTE \\ DE LA QUERELLE DANS DEUX \\ DIALOGUES DE JEAN CHAPELAIN \\ ET JEAN-FRANÇOIS SARASIN
}

1638. Après plusieurs mois de querelle autour du Cid de Corneille et la publication de dizaines de textes ${ }^{1}$, paraissent Les Sentiments de l'Académie française sur la tragi-comédie du Cid, texte commandé par Richelieu et rédigé pour une grande part par Jean Chapelain, censé mettre fin au débat et prononcer un jugement. Avant même de discuter la pièce et les critiques dont elle a fait l'objet, ce texte se prononce sur la légitimité de la querelle elle-même. Est-il utile et bon de débattre des lettres, et à quelles conditions ?

Il est à souhaiter que sur des propositions indécises il naisse des contestations honnêtes, dont la chaleur découvre en peu de temps, ce qu'une froide recherche n'aurait pu découvrir en plusieurs années ; que l'entendement humain faisant un effort pour se délivrer de l'inquiétude des doutes, s'acquière promptement par l'agitation de la dispute, cet agréable repos qu'il trouve dans la certitude des connaissances. $^{2}$

La valeur heuristique de la querelle est ainsi soulignée. Mais cette valeur est soumise au respect d'une certaine éthique : la contestation doit être « honnête »

1 Sur cette querelle, voir H. Merlin, L'Excentricité académique. Littérature, institution, société, Paris, Belles-Lettres, 2001, chap. VII (« La querelle du Cid 》), p. 169-187 ; J.-M. Civardi, La Querelle du Cid (1637-1638) : édition critique intégrale, Paris, Champion, 2004.

2 J. Chapelain, Les Sentiments de l'Académie française sur la Tragi-Comédie du Cid, Paris, Camusat, 1638, p. 8-9. L'orthographe des citations a été modernisée. 


\section{Marine Roussillon}

et avoir pour objectif la connaissance de la vérité. Les rivaux doivent ainsi, malgré le conflit dans lequel ils sont engagés, poursuivre un but commun. Chapelain poursuit :

C'est une espèce de guerre qui est avantageuse pour tous, lorsqu'elle se fait civilement, que les armes empoisonnées y sont défendues. C'est une course, où celui qui emporte le prix semble ne l'avoir poursuivi que pour en faire un présent à son rival. ${ }^{3}$

Générosité, intérêt commun et civilité sont les conditions de la légitimité d'une dispute. S'élabore ainsi une définition paradoxale de la contestation, dans laquelle le désaccord recouvre un intérêt commun et le conflit est une modalité détournée de la coopération. Ce paradoxe, Chapelain le résume en une formule : ces querelles légitimes sont d'《agréables différends 》. Agréables : qui suscitent du plaisir, mais aussi qui font agréer, qui produisent de l'accord, du consentement. La querelle légitime est un différend qui produit du commun, un dissensus qui débouche sur du consensus. Les Sentiments de l'Académie cherchent ainsi à définir les conditions de légitimité du conflit littéraire, à la fois sur un plan éthique - en faisant la promotion de la civilité et de l'humilité - et sur un plan esthétique, en mettant en avant la recherche d'un plaisir partagé.

II s'agit ici d'observer la mise en œuvre de cette esthétique de l'« agréable différend » dans deux dialogues de la fin des années 1640 dans lesquels Jean Chapelain apparaît comme en personnage de « disputeur » et d'en saisir ainsi les enjeux. Le dialogue De la lecture des vieux romans ${ }^{4}$ est rédigé par Chapelain lui-même et mis en circulation de manière manuscrite en 1647. II met en scène trois interlocuteurs, Chapelain, Gilles Ménage et Jean-François Sarasin, qui débattent de l'intérêt de la lecture d'un roman médiéval, le Lancelot. Chapelain est surpris par ses amis en train de lire le roman. Il est attaqué par Ménage et se défend, secondé par Sarasin. Quelques mois plus tard, Sarasin publie, toujours de manière manuscrite, un second dialogue, intitulé S'il faut qu'un jeune homme soit amoureux ${ }^{5}$. Le texte se place explicitement dans la continuité du dialogue

3 Ibid.

4 Ce dialogue n'a pas été imprimé avant le XIXe siècle : J. Chapelain, De la lecture des vieuX romans, éd. A. Feillet, Paris, A. Aubry, 1870. F. Gégou en a proposé une édition critique à la suite de son édition de la Lettre-traité sur l'origine des romans de P.-D. Huet (Paris, Nizet, 1971). L'édition la plus récente de ce texte est celle de J.-P. Cavaillé : De la lecture des vieux romans, Paris, Paris-Zanzibar, 1999.

5 Vraisemblablement rédigé entre 1647 et 1649, ce dialogue est imprimé pour la première fois seulement dans l'éd. de 1656 des Euvres de Sarasin chez A. Courbé. Sur ce dialogue, voir

\section{2}


de Chapelain ${ }^{6}$ et met en scène les mêmes interlocuteurs, auxquels s'ajoute le personnage quasiment muet de M. de Trilport. Il s'agit cette fois de savoir si l'amour des dames est utile ou non à l'éducation des jeunes gens. Comme dans le premier dialogue, Chapelain est présenté comme un juge fiable, arbitre du bon goût? et Ménage défend la mauvaise cause. Sarasin, lui, hésite entre les positions de ses deux $a_{m i s}{ }^{8}$. Dans les deux cas, le dialogue prend la forme d'une dispute. L'échange se nourrit de la confrontation d'opinions différentes sur un même sujet (les romans médiévaux ou l'amour] et les interlocuteurs occupent des positions antagoniques. Le débat fait entendre deux voix dominantes : celle de Chapelain, défenseur des romans médiévaux et de l'amour, et celle de Ménage, qui critique l'un et l'autre. Les deux dialogues mobilisent donc un modèle agonique ${ }^{9}$ et proposent ainsi une réflexion éthique et esthétique sur la bonne manière de se quereller.

\section{Pourquoi se quereller ? Différend et dispute}

Les deux dialogues prennent pour point de départ une situation de désaccord, un différend: dans l'un, c'est la lecture de Chapelain qui suscite la désapprobation de Ménage; dans l'autre, c'est l'affirmation de Ménage selon laquelle l'amour des dames est inutile à la formation des jeunes gens qui provoque le désaccord des autres personnages. Dans De la lecture des vieux romans, le différend ne débouche cependant pas sur une véritable querelle. Ménage ne développe pas sa critique et se contente d'interroger ou de relancer Chapelain. Celui-ci ne semble développer son argumentation qu'à contre-cœur, comme si emporter la conviction de son auditoire équivalait à lui faire violence. II déclare ainsi préférer « laisser

J. Steigerwald, «"Galante Liebes-Ethik". Jean-François Sarasins Dialogue sur la question s'il faut qu'un jeune homme soit amoureux », dans K. Dickhaut et D. Rieger (éd.), Liebe und Emergenz. Neue Modelle der Affektbegreifens im französischen Kulturgedächtnis um 1700, Tübingen, Niemeyer, 2006, p. 33-54.

6 Le personnage de Chapelain évoque dans le dialogue « la grande conversation que nous eûmes il y a quelques mois », et le personnage de Trilport mentionne «le dialogue que vous [Chapelain] en avez composé » (J.-Fr. Sarasin, op. cit., p. 222).

7 On comparera avec la contribution de J. Steigerwald, où il est présenté de la même façon.

8 Pour une analyse plus précise des positions des différents interlocuteurs dans les deux dialogues, et de leurs implications quant à la posture des auteurs et aux prises de positions des acteurs dans le champ littéraire, voir C. Cazanave, Le Dialogue à l'âge classique, Paris, Champion, 2007, p. 198-213.

9 Je reprends ici une catégorie utilisée par C. Cazanave, op. cit., p. 81. 


\section{Marine Roussillon}

croire à M. Ménage ce qui lui plaira » que de «l'obliger à entendre [l'] apologie ${ }^{10}$ » du Lancelot. Le désaccord est présenté comme légitime, et la querelle comme désagréable et violente. Les refus réitérés d'argumenter de Chapelain contribuent à la construction d'un éthos humble et civil. Dans le même temps, ils affirment l'illégitimité de la dispute comme mode d'argumentation. Le différend légitime exclut le conflit, la polémique, la violence et privilégie une recherche de la vérité en coopération.

S'il faut qu'un jeune homme soit amoureux, au contraire, adopte la structure et le ton d'une véritable dispute. Ménage y expose longuement son opinion, puis Chapelain lui répond point par point. L'affrontement des deux partis débouche sur un jugement qui tranche la question. Mais cette fois-ci, la querelle ne repose pas sur un véritable différend; dès le début du dialogue, le désaccord entre les personnages est présenté comme fictif :

Mais soit que je ne sois pas de l'opinion de Monsieur Chapelain, moi à qui on reproche que je n'ai guère accoutumé d'être de celle des autres, soit que je me plaise à contester, comme vous me le dites souvent ; puis qu'il n'y a point ici de femmes, je ne vous dissimulerai pas que quelques avantages qu'on attende de l'attachement qu'on a pour elles, il s'y rencontre d'autre côté tant de choses qui peuvent empêcher qu'on ne devienne honnête homme, que je suis tout prêt de disputer contre la vieille thèse, qui expose qu'il est impossible qu'un homme soit fort propre pour le monde, s'il n'a été amoureux en sa jeunesse. Et moi, interrompit Monsieur Chapelain, je suis tout prêt de soutenir le contraire. ${ }^{11}$

L'union du groupe d'amis autour de valeurs qui font consensus n'est brisée que par jeu. La polémique est présentée comme un divertissement. Les deux dialogues mettent ainsi en œuvre une esthétique de l'agréable différend, soit en refusant la dispute au nom de l'agrément, soit au contraire en définissant la dispute comme une forme agréable dans la mesure où elle est gratuite et ne recouvre pas de désaccord réel. Dans un cas comme dans l'autre, la mise en scène d'un désaccord sert a contrario à affirmer le caractère illégitime du conflit violent et réel, en éthique comme en esthétique. Celui-ci n'est acceptable que s'il est réduit à un jeu ou s'il emprunte les formes du consensus. Ce rejet du conflit s'inscrit dans la revendication de cohésion du groupe présenté par ces textes : il s'agit de figurer l'union d'un groupe d'écrivains autour des valeurs partagées de la galanterie ${ }^{12}$. Mais dans le même temps, il participe de la construction d'une esthétique du

$10 \mathrm{~J}$. Chapelain, De la lecture des vieux romans, éd. J.-P. Cavaillé, p. 52.

$11 \mathrm{~J}$.-Fr. Sarasin, op. cit., p. 143-145.

12 Voir C. Cazanave, op. cit., p. 204 sq.

\section{4}


consensus, de l'agrément partagé, une esthétique de la paix qui stigmatise toute attitude polémique.

Dans ce cadre, la situation agonique mise en scène conserve cependant une valeur heuristique. L'échange contradictoire - même ludique ou atténué par l'humilité des intervenants - permet d'accéder plus rapidement à la vérité. Grâce à lui, une question peut être « véritablement bien agitée ${ }^{13}$ », comme l'écrit Chapelain dans la dédicace de son dialogue à Jean-François Paul de Gondi. La querelle n'est légitime que si elle est mise au service de cette recherche de vérité, de manière désintéressée. Dans De la lecture des vieux romans, le personnage de Chapelain s'excuse ainsi de défendre son point de vue en affirmant qu'il ne le fait que par générosité, parce que ses découvertes peuvent être utiles à son adversaire : «॥ sera même bien étonné et se sentira bien ingrat envers moi lorsqu'il apprendra que je ne m'y étais attaché que pour l'amour de lui ${ }^{14}$. » En revendiquant cet éthos de chercheur généreux, Chapelain s'inscrit dans la continuité des préceptes qu'il énonçait dans les Sentiments de l'Académie : « On peut même mériter de la louange en donnant du blâme, pourvu que les répréhensions partent du zèle de l'utilité commune, qu'on ne prétende pas élever sa réputation sur les ruines de celle d'autrui ${ }^{15}$. 》 La bonne querelle n'est donc pas un conflit, mais une recherche généreuse qui emprunte les formes de la contradiction. La valeur de la situation agonique mise en scène par les dialogues tient encore au plaisir que cette situation procure. Dans la dédicace de son dialogue, Chapelain affirme l'avoir écrit pour « donner le plaisir de cet entretien ${ }^{16}$ » à son dédicataire. Dans les dernières lignes du texte, les personnages de Sarasin et de Ménage quittent Chapelain « en riant $[\ldots]$, témoignant assez de satisfaction de l'entretien ${ }^{17} \gg$ qui vient de s'achever. L'esthétique de l'« agréable différend » mise en œuvre par les dialogues articule ainsi, d'une part, une promotion de l'échange contradictoire comme forme à la fois propre à la recherche de la vérité et agréable et, d'autre part, une condamnation du conflit comme violence désagréable et nuisible à la communauté.

$13 \mathrm{~J}$. Chapelain, De la lecture des vieux romans, éd. cit., p. 50.

14 lbid., p. 52.

$15 \mathrm{~J}$. Chapelain, Sentiments de l'Académie française sur la tragi-comédie du Cid, éd. cit., p. 5.

16 J. Chapelain, De la lecture des vieux romans, éd. cit., p. 50.

17 Ibid., p. 85. 


\section{Le refus du conflit : «entretien doux et aisé 》 versus « crierie querelleuse »}

Cette double exigence, heuristique et esthétique, fonde une esthétique de la querelle galante, que le personnage de Chapelain tente de définir dans S'il faut qu'un jeune homme soit amoureux :

Je m'accommoderai même à votre manière de philosopher, qui est sans doute, la plus propre pour la conversation, et de laquelle je me sers volontiers, quoiqu'elle ne soit pas si sévère que celle que nous pratiquons d'ordinaire, mais elle n'est pas moins forte pour être plus parée; et il vaut toujours mieux faire confesser volontairement qu'en mettant le poignard sur la gorge, ainsi donc, je continuerai de bannir de notre discours ces syllogismes de l'école qui donnent la migraine à ceux qui s'attachent à les comprendre ou à les résoudre, nous ne tirerons pas l'Amour d'entre les Grâces pour le mettre entre les bras de la Chicane ; et je donnerai bon ordre que notre entretien, qui jusques ici a été doux et aisé, ne dégénère pas en une crierie querelleuse de deux maîtres ès Arts. ${ }^{18}$

La dispute se voit vidée de sa dimension conflictuelle. Elle est une « conversation », un « entretien doux et aisé ». Le refus de la violence est explicite : «il vaut toujours mieux faire confesser volontairement qu'en mettant le poignard sous la gorge. » Le but de l'échange n'est pas la victoire, mais l'agrément : à la fois le plaisir (la conversation doit être «parée » et faire appel aux « Grâces ») et le consentement. Cette esthétique galante de la querelle est définie dans une opposition au modèle traditionnel de la dispute scholastique [«l'école 》) et de l'éloquence judiciaire (《la Chicane »), caractérisé quant à lui par la violence du conflit et par le désir individuel et égoïste de se faire valoir.

Ce contre-modèle de la dispute ou du procès est fréquemment convoqué dans les deux dialogues. Le vocabulaire juridique est employé par tous les personnages, aussi bien dans le dialogue de Chapelain que dans celui de Sarasin. Dans ce dernier, la dispute sur la valeur de l'amour se superpose même à une affaire judiciaire : le dialogue a lieu dans le moment où Sarasin attend la levée d'une accusation qui pèse sur lui ${ }^{19}$ et la réconciliation finale coïncide avec la proclamation de l'innocence du poète. Dans De la lecture des vieux romans, l'illégitimité de ce modèle fait

18 J.-Fr. Sarasin, op. cit., p. 193-194.

19 Au début du dialogue, Sarasin se présente comme en butte à des accusations qui l'ont poussé à renoncer à l'écriture. Cette situation n'est pas une fiction. En juillet 1647, Sarasin a été accusé d'avoir écrit des vers contre l'Orfeo de Luigi Rossi, joué le 2 mars 1647 avec le patronage éclatant de Mazarin, et il est emprisonné à la Bastille. L'enquête est confiée à Particelli d'Emeri et l'innocence de Sarasin finit par être reconnue. Mais c'est à la Bastille, et non chez son ami du Pille, comme il 
cependant consensus : tous les interlocuteurs partagent une esthétique commune de la conversation galante, refusant la pédanterie et le conflit. Dans S'il faut qu'un jeune homme soit amoureux, au contraire, la distinction entre l'esthétique galante de la contestation et le modèle érudit de la dispute est mise en scène à travers l'opposition entre la rhétorique de Ménage, proche du modèle érudit, et celle de Chapelain, plus mondaine. Ménage est accusé à plusieurs reprises de vouloir forcer la conviction de ses auditeurs, d'utiliser des arguments d'autorité et des exagérations pour leur faire violence. Trilport se plaint ainsi :

En vérité, si quelque chose me choque, c'est de voir que Monsieur Ménage nous traite comme des enfants, auxquels on montre des diables peints avec des ongles, des griffes, et un regard épouvantable, afin qu'ils en aient peur [...]. Ainsi Monsieur Ménage avec ces îles, ces peintures imaginaires, ces exemples fabuleux, tâche de nous épouvanter et de nous détourner de la solide raison. À quoi bon même parler d'Agamemnon ni d'Aristote, pour savoir si un jeune homme doit être amoureux ?20

Chapelain au contraire suscite le plaisir de ses interlocuteurs, Ménage y compris, au point que ce sont eux qui le prient de poursuivre son discours lorsqu'il souhaite s'interrompre.

La mise à distance du modèle de la querelle est d'abord esthétique : les « crieries querelleuses » sont désagréables et l'agrément est du côté d'une esthétique du loisir mondain, valorisant le naturel plutôt que les «syllogismes». Les dialogues se présentent comme les transcriptions de paroles prononcées dans la spontanéité de la conversation. Chapelain écrit ainsi à son dédicataire :

Je vous en rapporterai les propres paroles, comme elles furent proférées par chacun de nous, sans y apporter d'autre ornement que celui qu'elles doivent à leur matière et qui accompagne quelquefois la naïveté d'un discours où il n'y a rien de prémédité. ${ }^{21}$

Ce choix du naturel va de pair avec une posture éthique d'humilité et d'égalité entre les interlocuteurs. Aucun des personnages, au début des dialogues, n'est détenteur d'une autorité qui lui permettrait de juger le différend et de trancher les débats. Chapelain ouvre ainsi De la lecture des vieux romans par un passage dans lequel il loue ses deux amis et se déprécie. De la même manière, au début de S'il faut qu'un jeune homme soit amoureux, le personnage de Sarasin affirme

le prétend dans le dialogue, que le poète a dû attendre sa réhabilitation. Voir H. Prunières, L'Opéra italien avant Lulli, Paris, Champion, 1913, p. 143.

20 J.-Fr. Sarasin, op. cit., p. 178.

21 J. Chapelain, De la lecture des vieux romans, éd. cit., p. 50. 


\section{Marine Roussillon}

son incapacité à juger des matières amoureuses. Ménage au contraire vante ses compétences en la matière, et la suite du texte invalide cette prétention. Dans les deux dialogues, l'émergence progressive d'une figure d'arbitre, en la personne de Chapelain, apparaît alors comme le résultat d'un mérite personnel et non comme la réalisation d'une autorité pré-établie. La légitimité de l'arbitre n'en est que plus forte, d'autant que son arbitrage est mis en scène comme faisant consensus. À la fin du dialogue de Sarasin, tous les interlocuteurs « demeurent d'accord que rien n'est si nécessaire à un jeune homme pour devenir accompli, que de servir une honnête femme 22 .

Face au modèle de la querelle, se construisent alors d'autres modèles de la contestation galante. Les métaphores les plus souvent convoquées sont celles du discours amoureux et du duel ou du jeu guerrier. Dans les deux cas, la conflictualité acquiert une dimension ludique qui la désamorce pour en faire un modèle de coopération et un instrument de plaisir. Dans De la lecture des vieux romans, Ménage demande à Chapelain de lui faire part de ses arguments comme un amant prierait sa maîtresse :

Je vous en prie, suivit M. Ménage, et je vous en défie même si la prière ne suffit pas, afin que par bonté ou par dépit vous me fassiez la grâce que je désire et que je n'espère pas. ${ }^{23}$

Dans S'il faut qu'un jeune homme soit amoureux, les interlocuteurs du dialogue s'imaginent en chevaliers de romans :

- Ce n'est pas la première fois, reprit [Ménage], que vous vous êtes mis plusieurs contre moi, et que pour cela non seulement je n'ai pas fui, mais même je n'ai pas été vaincu. C'est pourquoi je me résous dorénavant, quand je proposerai quelque chose, d'imiter le Rodomont de l'Arioste, qui appelait les Paladins au combat, deux à deux, ou trois à trois, et de prendre pour ma devise, Horatio sol contra Toscana tutta.

- Ce que vous dites nous obligera aussi, répondit Monsieur de Trilport, nonobstant vos rodomontades, à imiter ces Paladins qui n'allaient jamais deux contre un [...]. Et parce que Monsieur Chapelain est celui qui a relevé le gage de défi que vous avez jeté, nous le laisserons entrer le premier en lice, et je ne doute point qu'il ne vous mène bientôt à outrance. ${ }^{24}$

L'échange contradictoire est alors bien un jeu, et il emprunte les formes du loisir mondain. Chapelain et Ménage commencent ainsi tous deux par une tentative

22 J.-Fr. Sarasin, op. cit., p. 257.

23 J. Chapelain, De la lecture des vieux romans, éd. cit., p. 53.

24 J.-Fr. Sarasin, op. cit., p. 144-145. 
d'expliquer l'allégorie de l'amour qui rappelle les jeux mondains décrits par Charles Sorel dans La Maison des jeux quelques années plus tôt ${ }^{25}$.

La mise en scène d'échanges contradictoires dans les deux dialogues sert donc une opération de distinction : le groupe galant met à distance le modèle de la dispute, et avec lui les valeurs de l'humanisme érudit et l'esthétique de l'éloquence judiciaire. II assimile ces valeurs à une culture du conflit et se revendique comme porteur d'une esthétique de l'agrément, c'est-à-dire du consensus et du plaisir. II oppose le modèle de la conversation mondaine, plaisante et pacifiée, à celui de la dispute.

\section{Le retour du conflit : mauvaise foi et prétérition}

Cependant, s'en tenir là et vanter l'idéal mondain et galant d'une République des lettres pacifiée ${ }^{26}$ reviendrait à prendre au mot les revendications portées par les textes, en ignorant leur action propre. En définissant les valeurs galantes comme l'objet d'un consensus et le support de relations pacifiées, les textes prennent position dans les conflits qui traversent le champ littéraire et cherchent à construire l'hégémonie de la galanterie, comme esthétique et comme groupe. Le travail de contextualisation des dialogues qui permet de replacer l'esthétique de la querelle galante dans le contexte polémique de son émergence a été fait ailleurs $^{27}$. J'en resterai ici à l'étude de la mise en scène de la contradiction dans les dialogues, et m'arrêterai sur deux passages révélateurs de la manière dont le conflit fait retour dans des textes qui cherchent à l'occulter. Ces passages laissent apercevoir, au-delà de la revendication de consensus, l'ailleurs d'une conflictualité rejetée hors de la conversation. Ainsi mise en perspective, l'esthétique galante du consensus apparaît comme l'outil d'une revendication éminemment polémique (et politique) d'hégémonie.

Dans le dialogue de Sarasin, l'affirmation de consensus coexiste avec la mise en scène ludique d'un désaccord. Le personnage de Ménage est construit de manière à être un membre de la communauté, adhérant aux mêmes valeurs que les autres, tout en portant une parole contradictoire. Sa posture ludique lui permet de jouer un rôle, d'incarner l'altérité qui en dehors de cette situation ludique ne saurait

25 Ch. Sorel, La Maison des jeux, Paris, N. de Sercy, 1642. Voir A. Genétiot, Poétique du loisir mondain, Paris, Champion, 1997, p. 226 sq.

26 Comme le fait par ex. M. Fumaroli, Le Genre des genres littéraires français : la conversation, Oxford, Clarendon Press, 1992.

27 Notamment par C. Cazanave, op. cit., p. 191 sq. et 198 sq. 


\section{Marine Roussillon}

prendre la parole dans le groupe. Par son usage de la raillerie et de la mauvaise foi, il se place à la frontière entre appartenance au groupe et exclusion du groupe, et incarne ainsi, pendant un temps du dialogue, une altérité dont il s'agit de triompher. Lorsque Ménage accuse Platon d'avoir séduit la femme de Socrate, Xantippe, Chapelain dévoile ainsi sa mauvaise foi :

Car pour ce qui regarde Xantippe, le «peut-être » que vous y avez mis fait assez voir que vous n'avez pas cru que ce fût celle de Socrate, et je ne puis même m'imaginer que votre érudition vous ayant mis au premier rang des illustres de votre siècle, et vous étant attaché avec un soin si laborieux et un si heureux succès sur le Diogène Laërce, je ne puis dis-je m'imaginer ; sinon que vous avez voulu ou vous jouer sur de mêmes noms, ou tenter la bonté de votre mémoire; car au reste vous savez que le temps et les autres circonstances détruisent cette galanterie de Platon, et il y a grande apparence que s'il en eût été soupçonné, nous en verrions quelque chose dans les livres de ses ennemis ; qui ne lui eussent pas pardonné cette faute. ${ }^{28}$

Ménage est porteur d'un double discours. Explicitement, il a accusé Platon et condamné l'amour. Mais la façon dont cette accusation est modalisée et l'incompétence qu'elle révèle doit alerter le lecteur averti : Ménage n'adhère pas à son propre discours. Dans un second temps, Chapelain explicite cette mauvaise foi de Ménage. Ce double discours crée une connivence entre les lecteurs avertis, capables de reconnaître l'ironie de Ménage et de s'amuser de son argumentation comme d'un jeu d'esprit. Cette mauvaise foi ironique ou ludique renforce et valorise la communauté de ceux qui la comprennent contre un extérieur non nommé, que Ménage caricature. Le consensus ainsi construit et revendiqué (Chapelain redit l'appartenance réelle de Ménage à la communauté] est bien un consensus « contre ». La mise en scène de la contradiction sert non pas à faire une place à l'autre, mais à le rejeter hors du cercle de ceux qui peuvent parler. La négation du conflit n'est pas tant une utopie ou un idéal qu'un outil dans la construction d'une hégémonie.

La mauvaise foi de Ménage révèle ainsi la dimension polémique de l'esthétique de l'« agréable différend », tout en en soulignant une des limites : son accusation « plus ingénieuse que véritable ${ }^{29}$ » révèle une contradiction entre plaisir et vérité. Le discours agréable peut se révéler mensonger et sa séduction peut se faire au détriment de la dimension heuristique de la querelle. La même tension est présente dans le dialogue de Chapelain. Le désir de plaire à ses interlocuteurs, de conserver

28 J.-Fr. Sarasin, op. cit., p. 220.

29 Ibid., p. 241. 
une position humble et d'éviter le conflit empêche le personnage de Chapelain d'aller au bout de son argument. Comme il l'explique, pour plaire, l'écrivain doit chercher «les choses dont la persuasion n'est pas difficile 》:

Parce que le plaisir n'est que selon la nature ou selon l'usage $[\ldots]$, pour faire acquiescer l'esprit à ce qu'on lui propose, il faut qu'il lui soit naturel ou connu ; l'inconnu et l'impossible, bien loin de le satisfaire, n'étant bons qu'à le révolter. ${ }^{30}$

Lorsque Chapelain veut faire émerger une idée nouvelle, il doit donc introduire un personnage fictif pour rompre le consensus sans mettre le groupe en danger. Lorsqu'il s'agit de comparer la galanterie de Lancelot et celle des contemporains, Chapelain commence par refuser de se prononcer :

C'est à vous de juger laquelle des deux galanteries est la plus obligeante pour les dames $[\ldots]$. Pour moi, je ne prononcerai point sur une question si délicate où le préjugé de la mode ne laisse point aux suffrages leur naturelle liberté. ${ }^{31}$

Puis il met en scène un contradicteur imaginaire, ce qui lui permet d'introduire de manière détournée une situation de conflit dans le dialogue. La querelle a finalement lieu, mais par prétérition :

En effet, j'aurais bien de la peine à répondre à celui qui, pour défendre [la galanterie de Lancelot], me représenterait combien noble est la galanterie qui prouve sa passion par la recherche des dangers, par du sang et par des victoires, et quel avantage elle a sur celle qui ne la prouve que par des coquetteries et des assiduités, ou, au plus, que par des collations, des musiques et des courses de bague. J'aurais bien de la peine à lui persuader que de beaux pas de danse valussent mieux que de bons coups d'épée, que de beaux dedans ou de belles courses fussent plus considérables que des joutes à fer émoulu, que des combats opiniâtrés jusqu'à la perte de la vie, que des présents de vaincus et de prisonniers. [...] Si je ne lui accordais qu'il aurait raison de préférer cette galanterie à la nôtre, je ne pourrais, au moins, empêcher de lui accorder qu'elle ne peut raisonnablement passer pour ridicule. ${ }^{32}$

Elle s'achève par la constitution d'un nouveau consensus entre les présents. Le conflit a permis de modifier leur opinion et de les faire adhérer à une idée nouvelle. La prétérition, quant à elle, a préservé l'unité du groupe.

- S'il m'en parlait, dit Sarasin, comme vous feignez qu'il pourrait faire, je lui répondrais comme vous dites que vous feriez, et il faudrait être le plus injuste du

30 J. Chapelain, De la lecture des vieux romans, éd. cit., p. 62.

31 Ibid., p. 83.

32 Ibid. 


\section{Marine Roussillon}

monde, si l'on n'écoutait la raison de tous côtés, et si l'on ne prononçait que sur le rapport de l'une des parties.

- II me semble, me dit M. Ménage, que je suis de votre opinion, et que cet article n'a pas été plus mal examiné que les autres. ${ }^{33}$

Le conflit apparaît comme le seul moyen d'aller contre la doxa et de modifier le contenu du consensus, mais il ne peut pas être assumé. Ce passage met au jour une contradiction de l'esthétique de l'« agréable différend »: le plaisir est conservateur et ne permet pas l'émergence de vérités nouvelles. Les objectifs heuristique et esthétique entrent ainsi en opposition. La condamnation du conflit débouche sur une esthétique conservatrice.

La réflexion galante sur la querelle se développe donc dans plusieurs directions. D'une part, les galants sont les héritiers du modèle rhétorique de la dispute et reconnaissent la valeur heuristique de l'échange contradictoire. D'autre part, ils s'opposent à ce modèle pour des raisons éthiques et esthétiques. Parce que la galanterie réunit des écrivains que l'on peut appeler des « nouveaux doctes ${ }^{34}$ » et des mondains, elle refuse l'exhibition d'érudition, assimilée à une argumentation violente, elle affirme l'égalité des interlocuteurs et refuse tout ce qui pourrait s'apparenter à une prise de pouvoir des uns sur les autres. Enfin, parce que les écrivains galants cherchent à se définir comme groupe, ils privilégient la revendication de valeurs communes et la représentation d'un consensus. La querelle galante apparaît alors comme une querelle impossible : le conflit ne peut s'introduire dans les textes que sous la forme ludique du jeu mondain. Ce refus du conflit au nom du plaisir partagé donne à l'esthétique galante une dimension politique qui sera en partie actualisée dans les années 1660, lorsque le pouvoir en fera l'esthétique officielle du royaume ${ }^{35}$. La promotion du plaisir permettra alors la glorification de la paix et de la soumission à un pouvoir agréable ${ }^{36}$.

Marine Roussillon

Groupe de Recherches Interdisciplinaires sur l'Histoire du Littéraire [Grihl]

33 Ibid.

34 Pour reprendre une expression d'A. Viala dans Naissance de l'écrivain, Paris, Éd. de Minuit, 1985, p. 33.

35 Voir A. Viala, La France galante, Paris, Puf, 2008, p. 85-90.

36 Voir sur ce sujet mon article « Amour chevaleresque, amour galant et discours politiques de l'amour dans Les Plaisirs de l'île enchantée (1664) », Littératures classiques, n 69, 2009, p. 65-78. 